\title{
111.
}

\section{ON A THEOREM OF M. LEJEUNE-DIRICHLET'S.}

[From the Cambridge and Dublin Mathematical Journal, vol. Ix. (1854), pp. 163-165.]

THE following formula,

$$
\Sigma q^{a x^{2}+2 b x y+c y^{2}}+\Sigma q^{a^{\prime} x^{2}+2 b x y+c^{\prime} y^{2}}+\ldots=2 \Sigma \delta^{\frac{1}{(n-1)}} \epsilon^{\epsilon^{\frac{1}{\left(n n^{2}-1\right)}}}\left(\frac{n}{P}\right) q^{n n^{\prime}},
$$

is given in Lejeune-Dirichlet's well-known memoir "Recherches sur diverses applications \&c." (Crelle, t. xxi. [1840] p. 8). The notation is as follows:-On the left-hand side $(a, b, c),\left(a^{\prime}, b^{\prime}, c^{\prime}\right), \ldots$ are a system of properly primitive forms to the negative determinant $D$ (i.e. a system of positive forms); $x, y$ are positive or negative integers including zero, such that in the sum $\Sigma q^{a x^{2}+2 b x y+c y^{2}}, a x^{2}+2 b x y+c y^{2}$ is prime to $2 D$, and similarly in the other sums; $q$ is indeterminate and the summations extend to the values first mentioned, of $x$ and $y$. On the right-hand side we have to consider the form of $D$, viz. we have $D=P S^{2}$ or else $D=2 P S^{2}$, where $S^{2}$ is the greatest square factor in $D$ and where $P$ is odd: this obviously defines $P$, and the values of $\delta, \epsilon$, which are always \pm 1 (or, as I prefer to express it, are always \pm ) are given as follows, viz.

$$
\begin{aligned}
& D=P S^{2}, \quad P \equiv 1(\bmod 4), \quad \delta, \quad \epsilon=++, \\
& D=P S^{2}, \quad P \equiv 3(\bmod 4), \quad \delta, \quad \epsilon=-+, \\
& D=2 P S^{2}, \quad P \equiv 1(\bmod 4), \quad \delta, \quad \epsilon=+- \\
& D=2 P S^{2}, \quad P \equiv 3(\bmod 4), \quad \delta, \quad \epsilon=-- \text {, }
\end{aligned}
$$

$n, n^{\prime}$ are any positive numbers prime to $2 D,\left(\frac{n}{P}\right)$ is Legendre's symbol as generalized by Jacobi, viz. in general if $p$ be a positive or negative prime not a factor of $n$, 
then $\left(\frac{n}{p}\right)=+$ or - according as $n$ is or is not a quadratic residue of $p$ (or, what is the same thing, $p$ being positive, $\left.\left(\frac{n}{ \pm p}\right) \equiv n^{\frac{1}{(n-1)}}(\bmod p)\right)$, and for $P=p p^{\prime} p^{\prime \prime} \ldots$,

$$
\left(\frac{n}{P}\right)=\left(\frac{n}{p}\right)\left(\frac{n}{p^{\prime}}\right)\left(\frac{n}{p^{\prime \prime}}\right) \ldots,
$$

and the summation extends to all the values of $n, n^{\prime}$ of the form above mentioned. In the particular case $D=-1$, it is necessary that the second side should be doubled. The method of reducing the equation is indicated in the memoir. The following are a few particular cases.

$D=-1$,

$$
\Sigma q^{x^{2}+y^{2}}=4 \Sigma(-)^{\frac{1}{2}(n-1)} q^{n n^{\prime}},
$$

or

$$
\begin{gathered}
\left(1+2 q^{4}+2 q^{16}+2 q^{36}+\ldots\right)\left(q+q^{9}+q^{25}+q^{49}+\ldots\right) \\
=\frac{q}{1-q^{2}}-\frac{q^{3}}{1-q^{6}}+\frac{q^{5}}{1-q^{10}}-\frac{q^{7}}{1-q^{14}}+\ldots \\
\Sigma q^{x^{2}+2 y^{2}}=2 \Sigma(-)^{\frac{1}{2}(n-1)+\frac{1}{8}\left(n^{2}-1\right)} q^{n n^{\prime}} \\
\left(1+2 q^{2}+2 q^{8}+2 q^{18} \ldots\right)\left(q+q^{9}+q^{25}+q^{49}+\ldots\right) \\
=\frac{q}{1-q^{2}}+\frac{q^{3}}{1-q^{6}}-\frac{q^{5}}{1-q^{10}}-\frac{q^{7}}{1-q^{14}}+\& c .
\end{gathered}
$$$$
D=-2,
$$

or

an example given in the memoir.

$$
\begin{gathered}
\begin{array}{c}
D=-3, \\
q^{x^{2}+3 y^{2}}=2 \Sigma\left(\frac{n}{3}\right) q^{n n^{\prime}}
\end{array} \\
\begin{aligned}
\text { or } \quad\left(q^{1}+q^{25}+q^{49}+q^{121}+q^{169} \ldots\right)(1+ & \left.+2 q^{12}+2 q^{18}+2 q^{108} \ldots\right) \\
& +2\left(q^{3}+q^{27}+q^{75}+q^{147}+\ldots\right)\left(q^{4}+q^{16}+q^{64}+q^{100} \ldots\right) \\
= & \frac{q+q^{5}}{1-q^{6}}-\frac{q^{5}+q^{25}}{1-q^{30}}+\frac{q^{7}+q^{35}}{1-q^{42}}-\frac{q^{11}+q^{55}}{1-q^{66}}+\ldots
\end{aligned}
\end{gathered}
$$

I am not aware that the above theorem is quoted or referred to in any subsequent memoir on Elliptic Functions, or on the class of series to which it relates: and the theorem is so distinct in its origin and form from all other theorems relating to the same class of series, and, independently of the researches in which it originates, so remarkable as a result, that I have thought it desirable to give a detached statement of it in this paper. 